\title{
FERMION ANALOGY FOR LAYERED SUPERCONDUCTING FILMS IN PARALLEL MAGNETIC FIELD
}

\author{
J. P. Rodriguez \\ Dept. of Physics and Astronomy, California State University, Los Angeles, CA 90032, USA \\ and Instituto de Ciencia de Materiales, Consejo Superior de Investigaciones Cientificas, \\ Universidad Autonoma de Madrid, Cantoblanco, 28049 Madrid, Spain.*
}

\begin{abstract}
The equivalence between the Lawrence-Doniach model for films of extreme type-II layered superconductors and a generalization of the back-scattering model for spin- $1 / 2$ electrons in one dimension is demonstrated. This fermion analogy is then exploited to obtain an anomalous $H_{\|}^{-1}$ tail for the parallel equilibrium magnetization of the minimal double layer case in the limit of high parallel magnetic fields $H_{\|}$for temperatures in the critical regime.
\end{abstract}

PACS Indices: 74.20.De, 74.20.Mn, 74.60.-w

* Present address. 
The advent of high-temperature superconductors has re-invigorated the study of layered superconductivity, ${ }^{1-3}$ wherein adjacent layers are Josephson coupled. An issue that remains open is the question of whether or not layer decoupling occurs in the presence of a parallel magnetic field. ${ }^{4-9}$ The standard phenomenological model used to study this problem theoretically is given by the Lawrence-Doniach (LD) free-energy functional, which in the absence of fluctuations of the magnetic field reads ${ }^{9}$

$$
E_{\mathrm{LD}}=J_{\|}\left[\sum_{l=1}^{N} \sum_{\vec{r}} \frac{1}{2}(\vec{\nabla} \theta)^{2}+\gamma_{*}^{\prime-2} \sum_{l=1}^{N-1} \sum_{\vec{r}}\left\{1-\cos \left[\theta(\vec{r}, l+1)-\theta(\vec{r}, l)-A_{z}(\vec{r}, l)\right]\right\}\right] .
$$

Here, $\theta(\vec{r}, l)$ denotes the phase of the superconducting order parameter, which lives on $N$ equally spaced square lattices with respective coordinates $l$ and $\vec{r}=(x, y)$, while the parallel magnetic field $B_{\|}=\left(\Phi_{0} / 2 \pi d\right) b_{\|}$directed along the $y$ axis is related to the vector potential above by $A_{z}=-b_{\|} x$, where $d$ represents the spacing between layers. Note that $\vec{\nabla}=\left(\Delta_{x}, \Delta_{y}\right)$, with $\Delta_{\mu} \theta(r)=\theta(r+\hat{\mu})-\theta(r)$. Also, $J_{\|}$is a measure of the in-plane phase rigidity, while $\gamma_{*}^{\prime}$ is directly related to the mass anisotropy parameter $\gamma=\left(m_{\perp} / m_{\|}\right)^{1 / 2}$ of the superfluid motion within $\left(m_{\|}\right)$and between $\left(m_{\perp}\right)$ planes. For the bulk case, $N \rightarrow \infty$, where magnetic screening effects must be included, it was first claimed by Efetov ${ }^{4}$ that layers decouple in the mixed phase for parallel fields $B_{\|}$above the characteristic scale $B_{*}^{\|} \sim \Phi_{0} / \gamma d^{2}$. Recent work, however, finds no evidence for a true phase transition as a function of field. ${ }^{6-9}$

The author has recently studied the relatively simpler problem of layered thin films of extreme type-II superconductors $\left(\lambda_{L} \rightarrow \infty\right)$ in parallel magnetic field. ${ }^{9}$ In this limit, magnetic screening effects are negligible and the problem reduces to the study of $N$ weakly coupled square-lattice $X Y$ models in the presence of uniform frustration; i.e., the thermodynamics is determined by the energy functional $E_{X Y}=-\sum_{r, \mu} J_{\mu} \cos \left[\Delta_{\mu} \phi(r)-A_{\mu}(r)\right]$, where $J_{x}=J_{\|}=J_{y}$ and $J_{z}=J_{\|} / \gamma^{\prime 2}$, with $\gamma^{\prime}=\gamma d / a$, and where $A_{\mu}=\left(0, b_{\perp} x,-b_{\|} x\right)$. Here $a$ denotes the square lattice constant. For thin enough films, $N d \ll \gamma^{\prime} d \ll \lambda_{L}$, in perpendicular magnetic fields, ${ }^{10} B_{\perp}=\left(\Phi_{0} / 2 \pi a\right) b_{\perp} \gg \Phi_{0} / \gamma^{2} d^{2}$, the thermodynamics of this model factorizes into parallel and perpendicular pieces that correspond respectively to Josephson vortices in between layers and to 2D perpendicular vortices within each layer. ${ }^{9}$ The latter is corroborated by Monte-Carlo simulation. ${ }^{11}$ Physically, this factorization is 
due to the fact that the modified Josephson penetration length $\gamma^{\prime} d$ sets the minimum perpendicular size for vortex loops that traverse many layers. ${ }^{12}$ The present factorization prevails in the presence of both parallel and perpendicular magnetic field as well, with the parallel thermodynamics determined by the following partition function for a layered Coulomb gas ensemble: ${ }^{9,13}$

$$
\begin{aligned}
Z_{\mathrm{CG}}=\sum_{\left\{n_{z}(\vec{r}, l)\right\}} \exp \left\{-\frac{1}{2 \beta_{\|}} \sum_{l=1}^{N} \sum_{\vec{r}, \vec{r}^{\prime}}\right. & \left.n_{z}(\vec{r}, l-1)-n_{z}(\vec{r}, l)\right] G^{(2)}\left(\vec{r}-\vec{r}^{\prime}\right)\left[n_{z}\left(\vec{r}^{\prime}, l-1\right)-n_{z}\left(\vec{r}^{\prime}, l\right)\right] \\
& \left.-i \sum_{l=1}^{N-1} \sum_{\vec{r}} n_{z}(\vec{r}, l) A_{z}(\vec{r}, l)-\frac{1}{2 \beta_{\perp}} \sum_{l=1}^{N-1} \sum_{\vec{r}} n_{z}^{2}(\vec{r}, l)\right\}
\end{aligned}
$$

where $n_{z}(\vec{r}, l)$ is an integer field over the layered structure that describes inter-layer fluxon excitations, ${ }^{14}$ with the fields at the boundary layers set to $n_{z}(\vec{r}, 0)=0=n_{z}(\vec{r}, N)$, and where $G^{(2)}=-\nabla^{-2}$ is the Greens function for the square lattice. Here, $\beta_{\|}=J_{\|} / k_{B} T$, while $\beta_{\perp}=\beta_{\|} / \gamma^{\prime 2}$. (Note that the assumption that magnetic screening be absent requires that the perpendicular component of the magnetic field exceed $\Phi_{0} / \lambda_{L}^{2}$.) By extending Polyakov's Hubbard-Stratonovich transformation of the neutral Coulomb gas in the plasma phase, ${ }^{15}$ it can further be shown ${ }^{9}$ that this layered Coulomb gas is equivalent to the LD model (1) at temperatures below the zero-field decoupling transition temperature, $T_{*}=$ $4 \pi J_{\|}$, in the limit of small fugacity, $y_{0}=\exp \left(-\gamma^{\prime 2} / 2 \beta_{\|}\right)$, where the effective anisotropy parameter is given by $\gamma_{*}^{\prime}=\left(\beta_{\|} / 2 y_{0}\right)^{1 / 2}$.

In this paper, we will first establish that the above layered Coulomb gas ensemble (2) is equivalent to a one-dimensional (1D) fermion analogy consisting of coupled chains. The latter is a generalization of the repulsive back-scattering model for spin- $1 / 2$ fermions introduced by Luther and Emery (LE) ${ }^{16}$ where the spin is identified with the layer index. The Hamiltonian for this model is divided into two parts, $H=H_{\|}+H_{\perp}$, with

$$
\begin{aligned}
H_{\|}= & \sum_{l=1}^{N} \sum_{k}\left\{v_{F} k\left[a^{\dagger}(k, l) a(k, l)-b^{\dagger}(k, l) b(k, l)\right]-\mu_{l}\left[a^{\dagger}(k, l) a(k, l)+b^{\dagger}(k, l) b(k, l)\right]\right\} \\
& +U_{\|} \sum_{l=1}^{N} \int d x \Psi_{L}^{\dagger}(x, l) \Psi_{R}^{\dagger}(x, l) \Psi_{L}(x, l) \Psi_{R}(x, l)
\end{aligned}
$$

and

$$
H_{\perp}=U_{\perp} \sum_{l=1}^{N-1} \int d x\left[\Psi_{L}^{\dagger}(x, l) \Psi_{R}^{\dagger}(x, l+1) \Psi_{L}(x, l+1) \Psi_{R}(x, l)+\text { H.c. }\right]
$$


and with field operators $\Psi_{R}(x, l)=L_{x}^{-1 / 2} \sum_{k} e^{i k x} a(k, l)$ and $\Psi_{L}(x, l)=L_{x}^{-1 / 2} \sum_{k} e^{i k x} b(k, l)$ for right $(R)$ and left $(L)$ moving fermions. We thus have $N$ Tomonaga-Luttinger chains, with adjacent chains coupled via a LE-type repulsive back-scattering interaction $\left(U_{\perp}>0\right)$. The magnetic flux between consecutive layers in the LD model (1) is given by $b_{\|}=$ $2 \pi\left(N_{l+1}-N_{l}\right) / L_{x}$, where $N_{l}$ denotes the number of spin-less fermions in the $l^{\text {th }}$ chain. The above fermion analogy thus completes a triad of equivalent descriptions (1), (2), and (3a,b) that generalize the known equivalences between the sine-Gordon model, the 2D Coulomb gas, and the massive Thirring/LE models to $N$ layers. ${ }^{14-18}$ By analyzing the double-layer case $(N=2)$ that corresponds to the original spin- $1 / 2$ back-scattering model, ${ }^{16}$ we find that the equilibrium magnetization for parallel fields shows an anomalous $B_{\|}^{-1}$ tail in the high-field limit at temperatures near $T_{*}$ (see Fig. 1), as opposed to the $B_{\|}^{-3}$ tail expected from Ginzburg-Landau theory. ${ }^{5,9}$ This is a result of the entropic presure between neighboring Josephson vortices, which the fermion analogy correctly accounts for. Nevertheless, we continue to obtain at best only a cross-over behavior as a function of parallel magnetic field (see Fig. 1), which had been claimed earlier on the basis of a semi-classical analysis. ${ }^{9}$

Equivalence. We now proceed to show that the above 1D fermion back-scattering model is equivalent to the layered Coulomb gas ensemble (2) by extending the demonstration given by Chui and $\mathrm{Lee}^{17}$ for the minimal case $N=2$. The $S$ matrix, which measures the overlap of the unperturbed $\left(U_{\perp}=0\right)$ groundstate $\Phi_{0}$ with the exact one $\Psi_{0}$, is $\left\langle\Phi_{0} \mid \Psi_{0}\right\rangle=\left\langle\Phi_{0}\left|\exp \left(-i \bar{T}_{0} H_{\perp}\right)\right| \Phi_{0}\right\rangle$. After making the standard canonical transformation to trivialize the $U_{\|}$term in (3a) and employing the boson representation for the back-scattering interaction, ${ }^{16,19} H_{\perp}$, a perturbative expansion of the former exponential yields

$$
\begin{aligned}
\left\langle\Phi_{0} \mid \Psi_{0}\right\rangle= & \sum_{n=0}^{\infty}\left[U_{\perp} /(2 \pi \alpha)^{2}\right]^{2 n} \int_{0}^{i \bar{T}_{0}} d \tau_{2 n} \ldots \int_{0}^{\tau_{3}} d \tau_{2} \int_{0}^{\tau_{2}} d \tau_{1}\left(\Pi_{i} \int_{0}^{L_{x}} d x_{i}\right) \\
& \times \sum_{\left\{n_{z}(i)\right\}}\left\{\left\langle\Pi_{i} \exp \left\{n_{z}\left(x_{i}, \tau_{i}, l_{i}\right) e^{\phi}\left[\phi_{L}\left(x_{i}, \tau_{i}, l_{i}\right)+\phi_{R}\left(x_{i}, \tau_{i}, l_{i}\right)\right]\right\}\right\rangle_{0}\right. \\
& \left.\times \Pi_{i} \exp \left[-i n_{z}\left(x_{i}, \tau_{i}, l_{i}\right) A_{z}\left(x_{i}, \tau_{i}, l_{i}\right)\right]\right\}
\end{aligned}
$$

where $n_{z}\left(x_{i}, \tau_{i}, l_{i}\right)= \pm 1$ are inter-layer fluxon charge distributions ${ }^{13}$ such that $\sum_{i} n_{z}\left(x_{i}, \tau_{i}, l_{i}\right)=$ 0 , where $A_{z}(x, \tau, l)=-2\left(k_{F, l+1}-k_{F, l}\right) x$ is a function of the Fermi wavenumbers, $k_{F, l}$ 
corresponding to each chain, $l$, and where the average $\langle\ldots\rangle_{0}$ is over the non-interacting groundstate. Above, $\phi_{j}(x, \tau, l)=\psi_{j}(x, \tau, l+1)-\psi_{j}(x, \tau, l)$, where $\psi_{j}(x, \tau, l)$ is the time evolution of the local operator $\psi_{j}(x, l)=\lim _{\alpha \rightarrow 0} 2 \pi L_{x}^{-1} \sum_{k} k^{-1} \exp \left(-\frac{1}{2} \alpha|k|-i k x\right) \rho_{j}(k, l)$ that results from the boson representation, with the usual particle-hole operators given by $\rho_{R}(k, l)=\sum_{q} a^{\dagger}(q+k, l) a(q, l)$ and $\rho_{L}(k, l)=\sum_{q} b^{\dagger}(q+k, l) b(q, l)$. Notice that $n_{z}=1$ corresponds to the choice of the step-up term for $H_{\perp}$ in the perturbative expansion, while $n_{z}=-1$ corresponds to the step-down term. Also, we have that $\tanh 2 \phi=U_{\|} / 2 \pi v_{F}$, as a result of the above-mentioned canonical transformation, ${ }^{16}$ which yields a renormalized Fermi velocity equal to $v_{F}^{\prime}=v_{F} \operatorname{sech} 2 \phi$. After separating $\psi_{j}$ into creation and destruction pieces, changing variables to $y=v_{F}^{\prime} \tau$ and $L_{y}=v_{F}^{\prime} i \bar{T}_{0}$, and extending the Chui and Lee procedure ${ }^{17}$ to the present case (4), we obtain the equality $\left\langle\Phi_{0} \mid \Psi_{0}\right\rangle=Z_{\mathrm{CG}}$ along with the following identifications:

$$
\begin{aligned}
b_{\|} & =2\left(k_{F, l+1}-k_{F, l}\right), \\
\beta_{\|}^{-1} & =4 \pi e^{2 \phi}, \\
y_{0} & =(2 \pi)^{-2}(a / \alpha)^{2}\left(\left|U_{\perp}\right| / v_{F}^{\prime}\right) .
\end{aligned}
$$

Notably, since $N_{l}=\pi^{-1} k_{F, l} L_{x}$ gives the number of spinless fermions in a given chain, we obtain the relationship $b_{\|}=2 \pi\left(N_{l+1}-N_{l}\right) / L_{x}$ for the average magnetic induction in between layers $l$ and $l+1$ announced in the introduction. Notice that the back-scattering term $\left(U_{\perp}\right)$ sets the anisotropy parameter, $\gamma_{*}^{\prime}$, in the LD model (1), whereas the trivial intra-chain interaction $\left(U_{\|}\right)$sets the temperature. Finally, if we approximate the $S$ matrix of the fermion analogy by $\left\langle\Phi_{0} \mid \Psi_{0}\right\rangle=\exp \left\{-i \bar{T}_{0}\left[E_{F}\left(U_{\perp}\right)-E_{F}(0)\right]\right\}$, where $E_{F}\left(U_{\perp}\right)$ denotes the energy of the groundstate $\Psi_{0}$, then we can identify the free energy $G_{s}-G_{n}$ of the LD model in parallel field (1) with the latter via

$$
\left(L_{y} / v_{F}^{\prime}\right)\left[E_{F}\left(U_{\perp}\right)-E_{F}(0)\right]=\left(G_{s}-G_{n}\right) / k_{B} T .
$$

Thermodynamic properties of the layered superconductor are in this way directly related to ground-state properties of the fermion analogy.

Double Layer. Consider now the minimal $N=2$ case, where the LD model (1) reduces to the sine-Gordon model, the ensemble (2) reduces to the conventional 2D Coulomb gas, and where the fermion analogy $(3 \mathrm{a}, \mathrm{b})$ is simply the original LE back-scattering 
model for spin- $1 / 2$ fermions. In this instance, charge and spin particle-hole operators $\rho_{j}^{\prime}(k)=2^{-1 / 2}\left[\rho_{j}^{\prime}(k, 1)+\rho_{j}^{\prime}(k, 2)\right]$ and $\sigma_{j}^{\prime}(k)=2^{-1 / 2}\left[\rho_{j}^{\prime}(k, 2)-\rho_{j}^{\prime}(k, 1)\right]$ can be defined, which separate out of the canonically transformed Hamiltonian $H_{\|}^{\prime}$. Furthermore, at the special point $2^{1 / 2} e^{\phi}=1$ identified by LE, the spin part becomes equivalent to the one-body Hamiltonian

$$
H_{\sigma}^{\prime}=v_{F}^{\prime} \sum_{k} k\left(a_{k}^{\dagger} a_{k}-b_{k}^{\dagger} b_{k}\right)+\Delta_{\sigma} \sum_{k}\left(a_{k}^{\dagger} b_{k}+\text { H.c. }\right)-2^{1 / 2} \mu \sum_{k}\left(a_{k}^{\dagger} a_{k}+b_{k}^{\dagger} b_{k}\right)
$$

for spinless fermions, where the spin particle-hole operators are given by $\sigma_{R}^{\prime}(k)=\sum_{q} a_{k+q}^{\dagger} a_{q}$ and $\sigma_{L}^{\prime}(k)=\sum_{q} b_{k+q}^{\dagger} b_{q}$. Above, $\Delta_{\sigma}=U_{\perp}(2 \pi \alpha)^{-1}$ is the spin gap, while $\mu=\frac{1}{2}\left(\mu_{2}-\mu_{1}\right)$ is the external field. We therefore have energy eigenvalues $\varepsilon_{k}= \pm\left(v_{F}^{\prime 2} k^{2}+\Delta_{\sigma}^{2}\right)^{1 / 2}$ for such fermions. Notice that the procedure followed here is to first obtain the spinless fermion Hamiltonian in the absence of field, and to then add the trivial chemical potential shift in the presence of field. Let us begin by computing the lower-critical field of the double layer superconductor, and hence turn off the external field within the fermion model $(\mu=0)$. The edges of the double layer contribute Dirac Eq.-type bound states at zero energy that decay as $e^{ \pm x / \lambda_{J}}$, where $\lambda_{J}=v_{F}^{\prime} /\left|\Delta_{\sigma}\right|$ is the effective Josephson penetration length. ${ }^{20}$ Then Eq. (8) implies that the line-tension of a single Josephson vortex is given by $\varepsilon_{\|}=k_{B} T\left|\Delta_{\sigma}\right| / v_{F}^{\prime}=2 \pi J_{\|} / \lambda_{J}$, since $\left|\Delta_{\sigma}\right|$ gives the energy cost of adding one fermion/flux quantum to the system, and since $k_{B} T=2 \pi J_{\|}$at this special point [see Eq. (6)]. The lower-critical field is then simply $H_{c 1}^{\|}=4 \pi \varepsilon_{\|} / \Phi_{0}$. Last, we note that the identification (7) implies that the bare Josephson penetration length corresponding to the LD model (1) is equal to $\gamma_{*}^{\prime} a=\left(\pi v_{F}^{\prime} /\left|U_{\perp}\right|\right)^{1 / 2} \alpha$ at the present temperature, while $\lambda_{J}=2 \pi\left(v_{F}^{\prime} /\left|U_{\perp}\right|\right) \alpha$. In the limit of weak coupling, $U_{\perp} \rightarrow 0$, the latter renormalized Josephson penetration length is then much larger than the corresponding bare scale. In particular, the ratio of the parallel lower-critical field to the bare one set by the LD functional is equal to $\gamma_{*}^{\prime} a / \lambda_{J} \sim \alpha / a \gamma_{*}^{\prime}$, which is small if $\alpha \sim a$. This effect is a result of vortex wandering. ${ }^{9,22}$

To compute the parallel equilibrium magnetization deep inside the mixed phase, $B_{\|} \gg$ $H_{c 1}^{\|}$, we first relate the external field $\mu$ to the parallel magnetic induction in the absence of inter-chain/layer coupling $\left(U_{\perp}=0\right)$; i.e., Pauli paramagnetism gives $b_{\|}=2 \pi\left(N_{2}-\right.$ $\left.N_{1}\right) / L_{x}=2 \pi \chi_{0} \mu$, where $\chi_{0}=4\left(2 \pi v_{F}^{\prime}\right)^{-1}$. Since the magnetization is generally given by $M_{\|}=-\frac{\partial}{\partial H_{\|}}\left[\left(G_{s}-G_{n}\right) / L_{x} L_{y} d\right]$, where $H_{\|}=B_{\|}-4 \pi M_{\|}=\left(\Phi_{0} / 2 \pi d\right) h_{\|}$is the magnetic 
field, we have by Eq. (8) that

$$
-4 \pi M_{\|}=\left(4 \pi / \Phi_{0}\right)\left(2 \pi k_{B} T / v_{F}^{\prime}\right)\left(\partial \mu / \partial h_{\|}\right) \frac{\partial}{\partial \mu}\left\{\left[E_{F}\left(U_{\perp}\right)-E_{F}(0)\right] / L_{x}\right\} .
$$

But $\partial E_{F} / \partial \mu=-\left(N_{2}-N_{1}\right)=-L_{x} \chi_{0}\left(\mu^{2}-\frac{1}{2} \Delta_{\sigma}^{2}\right)^{1 / 2}$, where we have used $N_{2}-N_{1}=$ $2^{1 / 2} k_{F} L_{x} / \pi$ in conjunction with $2^{1 / 2} \mu=\varepsilon_{k_{F}}$. If we presume that $\partial \mu / \partial h_{\|}$above is given by $\partial \mu / \partial b_{\|}$in the absence of inter-layer coupling, we obtain

$$
-4 \pi M_{\|}=2^{-1 / 2} H_{c 1}^{\|}\left[\left(1+\frac{B_{\|}^{2}}{B_{*}^{2}}\right)^{1 / 2}-\frac{B_{\|}}{B_{*}}\right]
$$

for the equilibrium magnetization, ${ }^{21}$ where $B_{*}=\left(2^{1 / 2} / \pi\right)\left(\Phi_{0} / \lambda_{J} d\right)$ is the parallel crossover field. [The ratio of this cross-over scale to the bare one $B_{0} \sim \Phi_{0} / \gamma_{*}^{\prime} a d$ set by the LD functional is again $B_{*} / B_{0} \sim \alpha / a \gamma_{*}^{\prime}$, and hence small if $\alpha \sim a$.] This simple functional form is plotted in Fig. 1. Notably, (10) predicts an anomalous $-4 \pi M_{\|} / H_{c 1}^{\|}=2^{-3 / 2} B_{*} / B_{\|}$tail for relatively high fields $B_{\|}>B_{*}$. Straight forward minimization of the LD functional (1) in parallel magnetic field leads to a much weaker $B_{\|}^{-3}$ dependence at high fields, ${ }^{5,9}$ with a more pronounced cross-over at $B_{*}$. This indicates that the entropic pressure generated by fluctuations of the vortex array is not negligible at temperatures in the critical regime, which is defined by the relationship $k_{B} T \sim J_{\|}(T)$ (see ref. 9).

What, however, is the nature of the vortex array itself? Clearly, the mixed phase corresponds to a gapless Luttinger liquid in terms of the LE model. Voit has shown that algebraic spin-density wave (SDW) correlations in space dominate in such case for $U_{\perp}>$ $0 .{ }^{19}$ Hence, by the equivalence demonstrated above, this implies that the array of Josephson vortices displays algebraic long-range order transverse to the applied magnetic field. This physics is accurately captured by the SDW meanfield theory for $(3 a, b)$ characterized by the self-consistency equation $\chi_{l}=L_{x}^{-1} \sum_{k}\left\langle a^{\dagger}(k, l) b(k, l)\right\rangle$ and gap equation $\Delta_{l}=U_{\|} \chi_{l}+$ $U_{\perp}\left(\chi_{l+1}+\chi_{l-1}\right)$. A standard analysis of this theory ${ }^{23}$ for the case $N=2$ yields a phase diagram identical to that derived via renormalization group arguments; ${ }^{17}$ i.e., an SDW phase $\left(\chi_{2}=-\chi_{1}\right)$ exists for (repulsive) $U_{\perp}>U_{\|}$. Notice that this implies a leading dependence $k_{B} T_{*}=4 \pi J_{\|}\left[1+\frac{1}{4}\left(\alpha / a \gamma_{*}^{\prime}\right)^{2}\right]$ for the decoupling transition temperature as a function of the anisotropy parameter by Eqs. (6) and (7). If an external field $|\mu|>\left|\Delta_{l}\right|$ is introduced, however, a unitarity catastrophe occurs, wherein $\left|\Delta_{l}\right|$ acquires an imaginary part. $^{23}$ This we take to be a signal of the Luttinger liquid $\left(k_{F} \neq 0\right)$ "instability". 
$N$ Layers. Given that the above meanfield theory works quite well for the minimal case $N=2$, it should be accurate at least with respect to the phase diagram for general $N$. For large $N$, an SDW phase $\chi_{l+1}=-\chi_{l}$ is stable for (repulsive) $U_{\perp}>\frac{1}{2} U_{\|} \cdot{ }^{23}$ We therefore argue by comparison with the match in the case of $N=2$ that an anomalous $B_{\|}^{-1}$ tail appears in the equilibrium parallel magnetization as well at high fields $B_{\|}>B_{*}$. And that the lower-critical field is given by the double-layer result as well can be shown by observing that flux penetration must begin at the edges, each of which reduce to a double layer at low fields and for weak inter-layer coupling. The latter can be seen via the Coulomb gas analogy (2), where it becomes obvious that the effect of neighboring layers is to produce a weak dielectric renormalization in the effective $2 \mathrm{D}$ Coulomb gas that resides at the edge in the limit of weak inter-layer coupling. ${ }^{22}$

In summary, we have obtained a fermion analogy (3) for extreme type-II layered superconductors in parallel magnetic field (1). In the minimal double-layer case, we predict an anomalous $H_{\|}^{-1}$ tail for the parallel magnetization in the limit of high parallel magnetic field, $H_{\|}$, for temperatures in the critical regime. A mean-field treatment of the fermion analogy for the $N$-layer case (3), however, suggests that this dependence is generic to extreme thin-film geometries. ${ }^{23}$

The author is indebted to E. Rezayi for discussions. He also is grateful for the hospitality of the Theoretical Division at Los Alamos National Laboratory, where this work originated. This work was supported in part by National Science Foundation grant DMR9322427 and the Spanish Ministry for Science and Education. 


\section{References}

1. W.E. Lawrence and S. Doniach, in Proceedings of the 12 $2^{\text {th }}$ International Conference on Low Temperature Physics (Kyoto, 1970), edited by E. Kanda (Keigaku, Tokyo, 1971) p. 361; see also M. Tinkham, Physica C 235, 3 (1994).

2. L.N. Bulaevskii, Zh. Eksp. Teor. Fiz. 64, 2241 [Sov. Phys. JETP 37, 1133 (1973)].

3. G. Blatter, M.V. Feigel'man, V.B. Geshkenbein, A.I. Larkin, and V.M. Vinokur, Rev. Mod. Phys. 66, 1125 (1994).

4. K.B. Efetov, Zh. Eksp. Teor. Fiz. 76, 1781 (1979) [Sov. Phys. JETP 49, 905 (1979)].

5. L. Bulaevskii and J.R. Clem, Phys. Rev. B 44, 10234 (1991).

6. L.V. Mikheev and E.B. Kolomeisky, Phys. Rev. B 43, 10431 (1991) (Both this ref. and the following one discusses an analogy for the LD model in terms of fermions that exist in between consecutive layers, which is contrary to the present work where the fermions live on each layer).

7. B. Horovitz, Phys. Rev. Lett. 72, 1569 (1994); Phys. Rev. B 47, 5964 (1993).

8. S.E. Korshunov and A.I. Larkin, Phys. Rev. B 46, 6395 (1992).

9. J.P. Rodriguez, "On the Decoupling of Layered Superconducting Films in Parallel Magnetic Field", Los Alamos preprint LA-UR-95-1908 (cond-mat/9604182).

10. L.I. Glazman and A.E. Koshelev, Phys. Rev. B 43, 2835 (1991).

11. Y.H. Li and S. Teitel, Phys. Rev. B 47, 359 (1993); ibid 49, 4136 (1994).

12. S. Hikami and T. Tsuneto, Prog. Theor. Phys. 63, 387 (1980); B. Chattopadyay and S. R. Shenoy, Phys. Rev. Lett. 72, 400 (1994).

13. J.P. Rodriguez, Europhys. Lett. 31, 479 (1995).

14. S.E. Korshunov, Europhys. Lett. 11, 757 (1990); G. Carneiro, Physica C 183, 360 (1991).

15. A.M. Polyakov, Nucl. Phys. B120, 429 (1977); Gauge Fields and Strings (Harwood, New York, 1987).

16. A. Luther and V.J. Emery, Phys. Rev. Lett. 33, 589 (1974); P.A. Lee, Phys. Rev. Lett. 34, 1247 (1975).

17. S.T. Chui and P.A. Lee, Phys. Rev. Lett. 35, 315 (1975).

18. S. Coleman, Phys. Rev. D 11, 2088 (1975).

19. J. Voit, Rep. Prog. Phys. 58, 977 (1995). 
20. M. Fabrizio and A.O. Gogolin, Phys. Rev. B 51, 17827 (1995).

21. A result similar to Eq. (10) was obtained long ago by V.L. Pokrovskii and A.L. Talanov, Zh. Eksp. Teor. Fiz. 78, 269 (1980) [Sov. Phys. JETP 51, 134 (1980)], for the case of $2 \mathrm{D}$ incommensurate crystals.

22. J.P. Rodriguez, to be published in Phys. Rev. B (cond-mat/9604152).

23. J.P. Rodriguez, unpublished. 


\section{Figure Captions}

Fig. 1. Shown is the equilibrium parallel magnetization characteristic of a double-layer superconductor deep inside the mixed phase $\left(B_{\|} \gg H_{c 1}^{\|}\right)$at temperatures in the critical regime. Notice that the pronounced $B_{\|}^{-1}$ tail resulting from entropic pressure practically destroys the decoupling cross-over at $B_{*}$ (compare with ref. 9). 


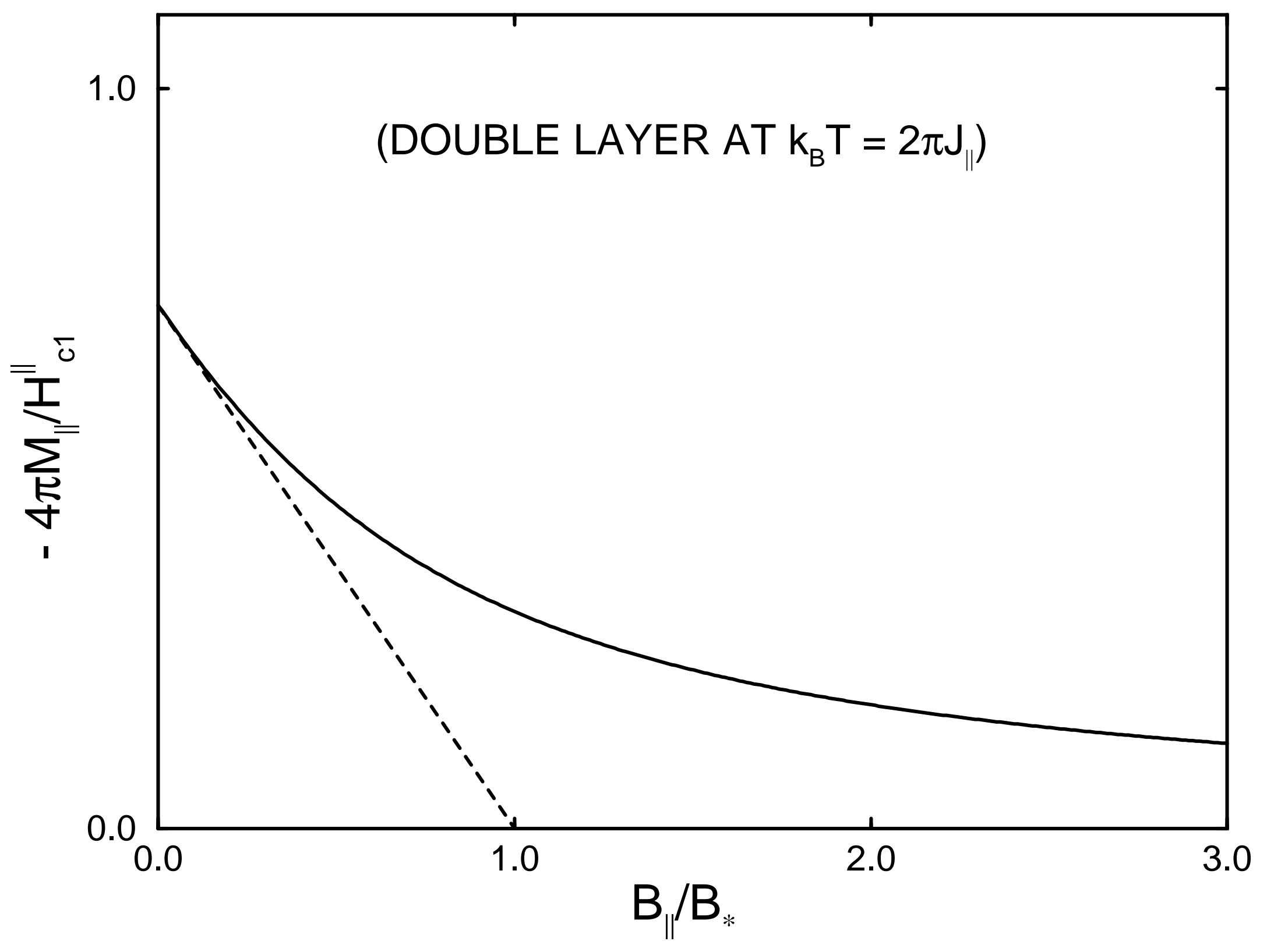

